\title{
An Unambiguous Distance-Based MIQP Model to Solve Economic Dispatch Problems with Disjoint Operating Zones
}

\author{
Mahdi Pourakbari-Kasmaei, Student Member, IEEE, Marcos J. Rider, Member, IEEE, and \\ Jose Roberto S. Mantovani, Member, IEEE
}

\begin{abstract}
This letter presents an unambiguous distance-based mixed-integer quadratic programming (UDB-MIQP) model to solve economic dispatch problems with disjoint operating zones. By considering the distances among the prohibited zones, the unnecessary variables will be eliminated and, consequently, a significant decrease, both in terms of variables and constraints, is obtained. The results show that the UDB model compared to existing MIQP models in the literature can achieve the global optimal solution with a significantly reduced problem complexity, both in terms of variables and constraints. In addition, the results indicate the superiority of the method in computational time reduction.
\end{abstract}

Index Terms-Distance based method, economic dispatch, mixed integer quadratic programming (MIQP), prohibited operating zone.

\section{INTRODUCTION}

$\mathbf{T}$ $\mathrm{HE}$ economic dispatch (ED) problem is considered the kernel of a power system, and its history dates back to the early 1920s [1]. In the literature, various mathematical models and optimization techniques such as classical, heuristic-based, and hybrid methods have been used to solve this problem [2]. First, a mixed integer quadratic programming (MIQP) model to solve ED problems with prohibited operating zones (POZ) was presented in [3]. To achieve this model for each unit with $k$ -1 POZs, $2 * k$ output variables were assigned, $k$ continuous variable for the feasible zones and $k$ binary variable to jump over POZs. Recently, a big-M based MIQP (M-MIQP) has been presented to address this drawback [4]. In this model, only $k$ -1 artificial binary variables via a binary coding are used. The M-MIQP model is simpler than the MIQP model and results in a decrease in constraints as well. However, two drawbacks of this model are: 1) defining a proper $M$ for each system; a very large $M$ yields serious numerical difficulties in a computer, and 2) complex binary codification. In this letter to address the aforementioned drawbacks, an unambiguous distance-based MIQP (UDB-MIQP) model, which has been adapted to the nature of commercial MIQP solvers to facilitate their pre-processing and probing techniques, is presented.

The mathematical formulation of the ED problem with POZs and ramp rate limits is as follows:

$$
\min \sum_{i=1}^{n_{g}} F_{i}\left(P_{i}\right)=\sum_{i=1}^{n_{g}} a_{i} P_{i}^{2}+b_{i} P_{i}+c_{i}
$$

Manuscript received July 02, 2014; revised October 21, 2014 and December 11, 2014; accepted December 29, 2014. Date of publication January 29, 2015; date of current version December 18, 2015. This work was supported by FAPESP No. 2011/13995-5, CNPq No. 305371/2012-6, and FEPISA. Paper no. TPWRS-00097-2014.

The authors are with the Universidade Estadual Paulista (UNESP), Ilha Solteira, Brazil (e-mail: mahdi.pourakbari@ieee.org).

Color versions of one or more of the figures in this paper are available online at http://ieeexplore.ieee.org.

Digital Object Identifier 10.1109/TPWRS.2015.2394317

$$
\begin{aligned}
& \text { s.t. } \\
& \sum_{i=1}^{n_{g}} P_{i}=P_{D} \\
& \max \left(\underline{P}_{i}, P_{i}^{0}-R D_{i}\right) \leq P_{i} \leq \min \left(\bar{P}_{i}, P_{i}^{0}+R U_{i}\right) \\
& \left\{\begin{array}{l}
\frac{P_{i}}{P_{i k}} \leq P_{i 1} \leq P_{i} \leq P_{i} \leq \bar{P}_{i 1}, \text { or } \\
\frac{P_{i z_{i}}}{P_{i}} \leq P_{i} \leq \bar{P}_{i z_{i}}=\bar{P}_{i}
\end{array}\right.
\end{aligned}
$$

where $i$ and $k$ denote the indexes of each generating unit and its operating zones, respectively; $z_{i}$ is the total number of operating (feasible) zones; $n_{g}$ is the total number of units; $F_{i}$ denotes the fuel cost of unit $i$ in quadratic form, $i=1, \ldots, n_{g} ; a_{i}, b_{i}$, and $c_{i}$ are the cost coefficients of unit $i ; P_{i}$ is the power generation of unit $i ; P_{i}$ and $\bar{P}_{i}$ are the lower and upper generation limits of unit $i$, and $P_{D}$ is the total demand; $P^{0}$ is the initial output power; and $R D_{i}$ and $R U_{i}$ are the ramp-down rate and ramp-up rate limits of unit $i$, respectively.

\section{Undisguised Distance-BASEd Method}

By modifying the POZ constraints (1d)-(2a), problem (1) becomes a distance-based MIQP problem:

$$
\begin{aligned}
& \underline{P_{i}}+u_{i 1} \underline{d_{i 1}}+\sum_{k=2}^{z_{i}-1} u_{i k} \underline{d_{i k}}+u_{i z_{i}} \underline{d_{i z_{i}}} \leq P_{i} \leq \bar{P}_{i 1}+u_{i 1} \bar{d}_{i 1} \\
& \quad+\sum_{k=2}^{z_{i}-1} u_{i k} \bar{d}_{i k}+u_{i z_{i}} \bar{d}_{i z_{i}} \\
& \sum_{k=1}^{z_{i}} u_{i k} \leq 1, \quad i=1, \ldots, n_{g}
\end{aligned}
$$

where $\underline{d}_{i k}$ and $\bar{d}_{i k}$ are the distances between the lower and upper limits of the first operating zone and the other operating zones of unit $i$, respectively; $u_{i k}$ is a decision-making binary variable that selects the appropriate $\underline{d}_{i k}$ and $\bar{d}_{i k}$ to jump over POZs.

In the proposed methodology, instead of a commonly used set of equality constraints that forces the units to operate in only one feasible zone, a set of inequality constraints (2b) is used. Replacing a set of equality constraints with the set of inequality constraints $(2 \mathrm{~b})$ results in fewer variables and reduces the complexity of the model because an equality constraint is considered a hard constraint. From Fig. 1, (2a), and the fact that a unit can only generate in one feasible zone at a time, $(2 b)$, it is clear that if the generation lies in the first feasible zone, all of the binary variables must be zero.

The main contribution of this letter is the proposal of a UDB-MIQP model that is adaptable with pre-solving and solving steps of all MIQP problems' solver. This adaptability results in a reduction of model complexity by eliminating unnecessary variables and constraints. The pre-solve step aims to reduce the size of the problem and improves the formulation via pre-processing and probing techniques, which rely mainly on the model's simplicity. During pre-processing, the identification of infeasibility, identification of redundancy, improving 


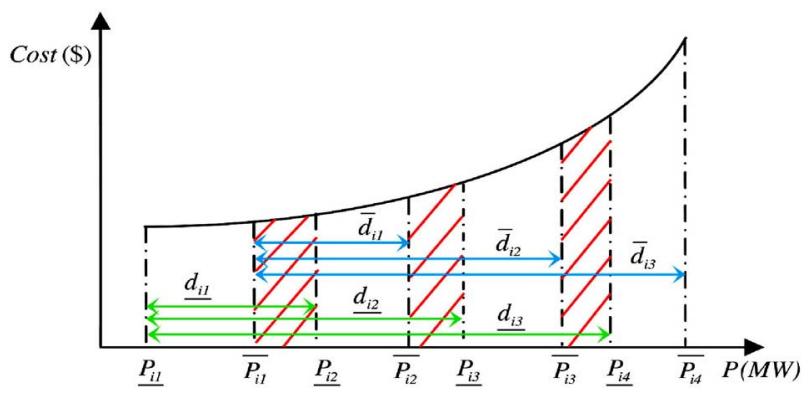

Fig. 1. Distances of operating zones' lower limits and upper limits from the first operating zone's lower limit and upper limit, respectively.

TABLE I

COMPARISON OF MIQP [3], M-MIQP [4], AND UDB-MIQP METHODS

\begin{tabular}{c|ccc|ccc}
\hline System & \multicolumn{3}{|c|}{ 15-Unit } & \multicolumn{3}{c}{ 40-Unit } \\
\hline Methods & MIQP & M-MIQP UDB-MIQP & MIQP & M-MIQP & UDB-MIQP \\
\hline \# of Vars. & 45 & 26 & 26 & 182 & 86 & 68 \\
\hline \# of Ctrs. & 69 & 60 & 13 & 273 & 194 & 51 \\
\hline \# of PE-POZ & 0 & 0 & 0 & 0 & 0 & 18 \\
\hline Cost (\$) & 32506.14 & 32506.14 & 32506.14 & 100767.68 & 100767.68 & 100767.68 \\
\hline Time (s, tick) & $(0.25,-)$ & $(0.03,-)$ & $(0.007,0.43)$ & $(0.186,-)$ & $(0.08,-)$ & $(0.009,1.03)$ \\
\hline PE-time(tick) & ---- & ---- & 0.17 & ---- & ---- & 0.68 \\
\hline ACT (p.u.) & 20.80 & 3.21 & 1.00 & 15.48 & 6.25 & 1.00 \\
\hline
\end{tabular}

the bounds, and rounding (for MIP) is considered while in probing, fixing the variables, improving the coefficients and the logical implications are taken into account. In [3], a complicated formulation for POZs has been used where the doubling of variables increases the difficulties in pre-processing and, on the other hand, two extra equality constraints make the fixing (probing) process more difficult. In M-MIQP, a complicated codification for binary variables has been used via an extra set of equality constraints that results in difficulties in pre-processing and probing techniques. The proposed UDB-MIQP model addresses the aforementioned difficulties in which it is not necessary to define an extra set of equality constraints, to use a complex codification, and even to find a proper auxiliary big M. In the proposed model, the distances determine how far the output power of a unit can go and, in this regard, the ramp rate may act as a catalyst that helps to eliminate the unnecessary binary variables and several related constraints. For example, in a unit with three POZs in Fig. 1, if $P_{i}^{0}=P_{i 1}$ and $R U_{i}$ is less than $d_{i 1}$, then based on $P_{i} \leq \min \left(\bar{P}_{i}, P_{i}^{0}+R U_{i}\right)$, the $P_{i}$ must be in the first operating zone and, consequently, the two other binary variables will be eliminated.

\section{CAse Studies AND Results}

To verify and demonstrate the effectiveness of the UDB-MIQP method, two commonly used test cases of a 15 -unit system [5] and 40-unit system [3] and a 2000-unit system as a large-scale system are conducted. To model the proposed method, a modeling language for mathematical programming (AMPL) is applied [6], while a commercial solver of CPLEX is used to solve this problem via a $2.67-\mathrm{GHz}$ computer with 3 GB of RAM.

Table I presents the number of variables (Vars), number of constraints (Ctrs), number of pre-eliminated POZs (PE-POZ), total CPU time, pre-elimination time (PE-time), and adjusted CPU time (ACT) [7]. It is worth mentioning that "tick" is independent from the processor speed and PC-clock and completely depends on the model's complexity. This may help in future studies. Because the other two studies did not report ticks, we use ACT to compare the times in a more efficient manner.

The optimal solution of the 15 -unit system is the same as those reported in the literature [3], [4], [8]. Although the number of variables for the proposed UDB-MIQP model is the same as [4], this model has 56 (or 93\%) and 47 (or 78\%) fewer constraints than the MIQP [3] and M-MIQP [4] models, respectively. The ACT shows that to obtain the same optimal solution, the UDB-MIQP model needs $0.048(1 / 20.8)$ and $0.31(1 / 3.21)$ of the time reported in [3] and [4], respectively.

The results of the 40-unit test system reveal that even with a significant elimination of variables and constraints as a result of using the UDB-MIQP model, the optimal solution is at hand, which shows a great advance. For instance, the UDB-MIQP model has 114 (or 68\%) and 18 (or 21\%) fewer variables than the MIQP [3] and M-MIQP [4] models, respectively. In this case, 18 pre-eliminated binary variables when considering the demands and ramp-rate limits are: $\mathrm{u}_{1}=0$ (for 10,28, and 29, which are units with one POZ), $\mathrm{u}_{2}=0$ (for 7, 24, 25, 26, and 27 , which are units with two POZs), and $\mathrm{u}_{1}=\mathrm{u}_{2}=0$ (for $13,14,15,16$, and 17, which are units with three POZs). The number of constraints for the UDB-MIQP model indicates 222 (or $81 \%$ ) and 143 (or 74\%) fewer constraints than the MIQP [3] and M-MIQP [4] models, respectively. The ACT for this system shows that to obtain the same optimal solution, the UDB-MIQP model needs only $0.16(1 / 6.25)$ and $0.064(1 / 15.48)$ of the time reported in [3] and [4], respectively.

Moreover, to show the effectiveness of the proposed model to solve large-scale systems, a 2000-unit test system, which contains fifty, 40-unit test systems, is considered. This system is considered under normal loading $(50 * 7000=350000 \mathrm{MW})$ and critical loading (449 $800 \mathrm{MW}$ ) conditions. The optimal solutions for normal and critical conditions are $\$ 5038384.36$ and $\$ 7596996.80$, respectively. The execution times are $0.16 \mathrm{~s}$ (134 tick) and $2.06 \mathrm{~s}$ (418 tick), respectively.

\section{CONCLUSIONS}

In this letter, a novel distance-based MIQP model (UDBMIQP) to solve the economic dispatch problems with disjoint operating zones is proposed. The UDB-MIQP model is a useful model for commercial solvers; during the pre-solve step of these solvers, this model gives them the capability of eliminating unnecessary artificial binary variables and several constraints. The results show that with the UDB-MIQP model, the complexity of the problem has been reduced significantly and also the rapidity of the model verifies its superiority over the existing models in the literature [3], [4], [8]. Additionally, the results verify the usefulness and effectiveness of the UDB-MIQP model to solve problems in large-scale systems.

\section{REFERENCES}

[1] H. H. Happ, "Optimal power dispatch-A comprehensive survey," IEEE Trans. Power App. Syst., vol. PAS-96, no. 3, pp. 841-854, 1977.

[2] M. Pourakbari-Kasmaei and M. Rashidi-Nejad, "An effortless hybrid method to solve economic load dispatch problem in power systems," Energy Convers. Manag., vol. 52, no. 8-9, pp. 2854-2860, 2011.

[3] L. G. Papageorgiou and E. S. Fraga, "A mixed integer quadratic programming formulation for the economic dispatch of generators with prohibited operating zones," Electr. Power Syst. Res., vol. 77, no. 10, pp. 1292-1296, 2007.

[4] T. Ding, R. Bo, W. Gu, and H. Sun, "Big-M based MIQP method for economic dispatch with disjoint prohibited zones," IEEE Trans. Power Syst., vol. 29, no. 2, pp. 976-977, Mar. 2014.

[5] S. O. Orero and M. R. Irving, "Economic dispatch of generators with prohibited operating zones: A genetic algorithm approach," Proc. Inst. Elect. Eng., Gener., Transm., Distrib., vol. 143, no. 6, pp. 529-534, Nov. 1996.

[6] R. Fourer, D. M. Gay, and B. W. Kernighan, AMPL: A Modeling Language for Mathematical Programming. Pacific Grove, CA, USA: Duxbury Press, 2002

[7] V. N. Dieu and W. Ongsakul, "Ramp rate constrained unit commitment by improved priority list and augmented Lagrange Hopfield network," Electr. Power Syst. Res., vol. 78, no. 3, pp. 291-301, 2008.

[8] R. A. Jabr, "Solution to economic dispatching with disjoint feasible regions via semidefinite programming," IEEE Trans. Power Syst., vol. 27, no. 1, pp. 572-573, Feb. 2012. 